\title{
Sequential Systemic Administrations of Combretastatin A4 Phosphate and Radioiodinated Hypericin Exert Synergistic Targeted Theranostic Effects with Prolonged Survival on SCID Mice Carrying Bifocal Tumor Xenografts
}

\author{
Junjie Li1,2, Marlein Miranda Cona ${ }^{1,2}$, Feng Chen ${ }^{1,2}$, Yuanbo Feng,1,2, Lin Zhou ${ }^{3}$, Guozhi Zhang1, Johan \\ Nuyts $^{3}$, Peter de Witte ${ }^{4}$, Jian Zhang 5 , Jie Yu1 ${ }^{1}$, Raymond Oyen ${ }^{6}$, Alfons Verbruggen ${ }^{4}$, Yicheng Ni1,2,5凶 \\ 1. Theranostic Laboratory, Department of Imaging \& Pathology, Faculty of Medicine, Biomedical Sciences Group, KU Leuven, Herestraat \\ 49, Leuven, Belgium. \\ 2. Molecular Small Animal Imaging Centre/MoSAIC, Biomedical Sciences Group, KU Leuven, Herestraat 49, Leuven, Belgium. \\ 3. Nuclear Medicine \& Molecular Imaging, Department of Imaging \& Pathology, Faculty of Medicine, Biomedical Sciences Group, KU \\ Leuven, Herestraat 49, Leuven, Belgium. \\ 4. Faculty of Pharmaceutical Sciences, Biomedical Sciences Group, KU Leuven, Herestraat 49, Leuven, Belgium. \\ 5. Laboratory of Translational Medicine, Jiangsu Academy of Traditional Chinese Medicine, Nanjing 210028, Jiangsu Province, China. \\ 6. Radiology Section, Department of Imaging \& Pathology, Faculty of Medicine, Biomedical Sciences Group, KU Leuven, Herestraat 49, \\ Leuven, Belgium.
}

\begin{abstract}
$\square$ Corresponding author: Prof. Yicheng Ni Address: Herestraat 49, BE-3000 Leuven, Belgium. Tel: +32-16-33 01 65, Fax: +32-16-34 37 65; E-mail: Yicheng.ni@med.kuleuven.be.
\end{abstract}

() Ivyspring International Publisher. This is an open-access article distributed under the terms of the Creative Commons License (http://creativecommons.org/ licenses/by-nc-nd/3.0/). Reproduction is permitted for personal, noncommercial use, provided that the article is in whole, unmodified, and properly cited.

Received: 2013.01.0I; Accepted: 2013.01.22; Published: 2013.02.13

\begin{abstract}
Objectives: Based on the soil-to-seeds principle, we explored the small-molecular sequential dual-targeting theranostic strategy (SMSDTTS) for prolonged survival and imaging detectability in a xenograft tumor model.

Materials and Methods: Thirty severe combined immunodeficiency (SCID) mice bearing bilateral radiation-induced fibrosarcoma-I (RIF-I) subcutaneously were divided into group A of SMSDTTS with sequential intravenous injections of combretastatin A4 phosphate (CA4P) and ${ }^{131} \mathrm{I}$-iodohypericin $\left({ }^{131} \mathrm{I}-\mathrm{Hyp}\right)$ at a $24 \mathrm{~h}$ interval; group $\mathrm{B}$ of single targeting control with CA4P and vehicle of ${ }^{131} \mathrm{I}-\mathrm{Hyp}$; and group $\mathrm{C}$ of vehicle control ( 10 mice per group). Tumoricidal events were monitored by in vivo magnetic resonance imaging (MRI) and planar gamma scintiscan, and validated by ex vivo autoradiography and histopathology. Besides, 9 mice received sequential intravenous injections of CA4P and ${ }^{131} \mathrm{I}-\mathrm{Hyp}$ were subjected to biodistribution analysis at 24, 72 and $120 \mathrm{~h}$.

Results: Gamma counting revealed fast clearance of ${ }^{|3|} \mathrm{I}$-Hyp from normal organs but intense accumulation in necrotic tumor over $120 \mathrm{~h}$. After only one treatment, significantly prolonged survival $(p<0.00 \mathrm{I})$ was found in group $A$ compared to group $B$ and $C$ with median survival of 33,22 , and 21 days respectively. Tumor volume on day 15 was $2.0 \pm 0.89,5.66 \pm 1.66$, and $5.02 \pm 1.0 \mathrm{~cm}^{3}$ with tumor doubling time $7.8 \pm 2.8,4.4 \pm 0.67$, and $4.5 \pm 0.5$ days respectively. SMSDTTS treated tumors were visualized as hot spots on gamma scintiscans, and necrosis over tumor ratio remained consistently high on MRI, autoradiography and histology.

Conclusion: The synergistic antitumor effects, multifocal targetability, simultaneous theranostic property, and good tolerance of the SMSDTTS were evident in this experiment, which warrants further development for preclinical and clinical applications.
\end{abstract}


Key words: vascular disrupting agents (VDAs), necrosis targeting radiotherapy, radiation-induced fibrosarcoma-1 (RIF-1), survival, synergistic antitumor effects.

\section{Introduction}

Cancerogenesis is a multistep process involving different mechanisms or pathways that lead to uncontrolled cell divisions, formation of malignancies, and eventually invasions or metastases. Such complexities make cancer cure biologically almost impossible. Development of metastasis depends on cross-talk between selected cancer cells (the seeds) and specific organ microenvironments (the soil) [1]. Instead of directly targeting cancer cells as widely elaborated, potential targets can also be cancer-related homeostatic factors, e.g., cancer microenvironment or stroma, which promote the survival, growth, angiogenesis, invasion and metastasis of tumor cells [2].

An alternative mode of cancer targeting namely a small-molecular sequential dual-targeting theragnostic strategy (SMSDTTS) has been proposed [3]. In this approach, the tumor vasculature and induced intratumoral necrosis are the two stable targets within the stroma domain of the cancer microenvironment (the soil), which can be selectively destroyed and further radioactively sterilized respectively by two small molecules, leading to indirect but more extensive and thorough killing of cancer cells (the seeds) [4-6]. The first target is the neovasculature characteristic of being relatively immature, irregularly shaped, proliferating, distended, hyperpermeable and disorganized in comparison to normal vasculature [7]. Vascular disrupting agents (VDAs) selectively damage such tumoral vasculature, particularly its capillary endothelium, causing vascular collapse and shutdown, depriving the tumor of blood and oxygen supply, and subsequently leading to rapid hemorrhagic necrosis inside the tumor [8]. After VDA treatment, layers of viable tumor cells always exist, which leads to unavoidable tumor relapse. However, the tumor necrosis induced by the VDA forms a secondary stroma target for the small molecular necrosis-avid compound (NAC) that is radiolabeled as a theranostic agent. As previously demonstrated, the NAC hypericin (Hyp) could be labeled with the tumouricidal radionuclide iodine-131 (131I) to form ${ }^{131}$ I-iodohypericin ( $\left.{ }^{131} \mathrm{I}-\mathrm{Hyp}\right)$, which may provide a solution for eradicating those residual tumor cells [4-6]. Upon systemic administration, both the extremely high necrosis avidity and the small molecular weight of ${ }^{131} \mathrm{I}-\mathrm{Hyp}$ facilitate its diffusion from well perfused region into necrotic tissue. Therefore, 131I-Hyp effectively delivers a high dose of radioactive "payload" to the necrotic stroma, irradiating or elim- inating the neighboring cancer cells and preventing tumor re-growth. Since the abnormal vasculature and inducible necrosis are two stromal targets universal to all solid malignancies, SMSDTTS may serve as a generic anticancer approach.

Previous animal data after one or two injections of single ${ }^{131} \mathrm{I}-\mathrm{Hyp}$ failed to show remarkable anticancer efficacy as compared with control groups $[4,9]$. This suggests that the presence of massive necrosis and minimum tumor residue should be prerequisites for successful anticancer effects of SMSDTTS. Preliminary efficacy of SMSDTTS has been evidenced in recent studies with rodents of hepatic allografts of rhabdomyosarcoma-1 (R1) [4-6], which however did not reveal potency of SMSDTTS on tumors of different origins and locations, as well survival data and long-term diagnostic potential, and therefore may not be strong enough to justify the hypothesis and conclusions for SMSDTTS. In this study we consecutively administered combretastatin A4 phosphate (CA4P) and ${ }^{131} \mathrm{I}-\mathrm{Hyp}$ in severe combined immunodeficiency (SCID) mice with bilateral subcutaneous xenografts of radiation-induced fibrosarcoma-1 (RIF-1), and explored the theranostic efficacy of SMSDTTS by evaluating the biodistribution of 131 I-Hyp, survival probability, in vivo imaging, ex vivo autoradiography, and histopathology of the tumor.

\section{Materials and Methods}

\section{Animals}

After approval by the institutional ethical committee for the use and care of laboratory animals, 40 male, 8-10 week old, SCID mice weighing 26-28 g were recruited for the experiments. Each mouse was kept in a separate cage with food and water provided ad libitum. Environmental conditions were maintained at a temperature of $22 \pm 2{ }^{\circ} \mathrm{C}$, a relative humidity of 60 $\pm 10 \%$, and an equal indoor light circle.

\section{Mouse bilateral RIF-I models}

RIF-1 tumor cells $\left(1 \times 10^{6}\right)$ were inoculated subcutaneously to the upper flank region of both sides in all SCID mice as xenograft tumor models to test the multifocal targetability or detectability of the SMSDTTS.

\section{Drug preparation and radiolabelling}

CA4P was purchased from Toronto Research 
Chemicals (www.trc-canada.com), diluted in Phosphate buffered saline (PBS) solution at a concentration of $5 \mathrm{mg} / \mathrm{ml}$, and iv injected at $10 \mathrm{mg} / \mathrm{kg}$. Hyp was commercially available (http://www.planta.at/ hyper/hyper.htm) with a purity $>98 \%$. Sodium iodide (Na131I) was supplied by MDS Nordion, Fleurus, Belgium. The specific activity was $7400 \mathrm{MBq} / \mathrm{ml}$, and the radionuclidic purity was $>99 \%$.

The Iodogen coating method was used for radioiodination of Hyp to form 131I-Hyp (Fig. 1). Briefly, by using an Iodogen (1,3,4,6-tetrachloro-3a, 6a-diphenylglycouril) tube (Thermo Scientific Pierce, Rockford, USA), radioiodination was conducted by adding $185 \mathrm{MBq}$ of $\mathrm{Na}^{131} \mathrm{I}, 50 \mu \mathrm{l}$ of $0.5 \mathrm{M}$ phosphate buffer solution ( $\mathrm{pH} 7.4$ ), and then $2 \times 10^{-6}$ mol of Hyp dissolved in $400 \mu$ l of dimethylsulfoxide. The mixture was incubated for 20 minutes, and the reaction was terminated by removal of the reaction mixture. A labeling yield of greater than $99.5 \%$ was determined with ascending paper chromatography using Whathman Filter paper grade No. 1 and $0.01 \mathrm{~N} \mathrm{HCl}$ as stationary and mobile phase respectively, resulting in a specific activity of ${ }^{131} \mathrm{I}-\mathrm{Hyp}$ of $185 \mathrm{MBq} / \mathrm{mg}$.
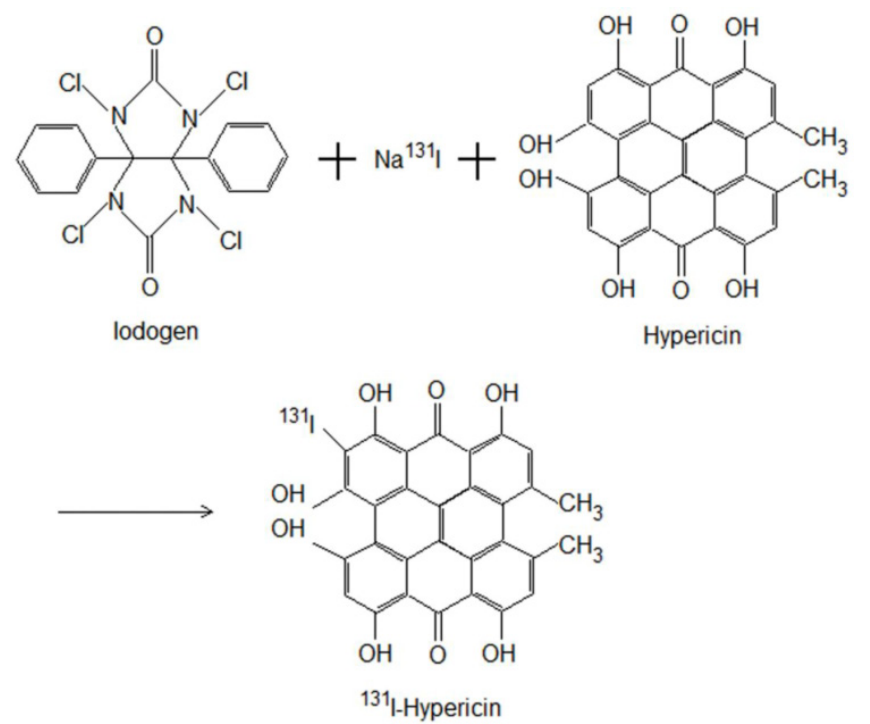

Figure I. Radioiodination of Hyp using lodogen as oxidizing agent to form |3||-Hyp.

\section{Experimental protocols}

The experiment began when the tumor diameter reached $0.8 \pm 0.2 \mathrm{~cm}$ at 14 days after implantation. As illustrated (Fig. 2), three subgroups of 3 mice having received sequential iv injections of CA4P $(10 \mathrm{mg} / \mathrm{kg})$ and ${ }^{131} \mathrm{I}-\mathrm{Hyp}(20 \mathrm{MBq} / \mathrm{kg}$ ) were sacrificed at 24, 72, or
$120 \mathrm{~h}$ for biodistribution analysis. For survival study, 30 mice with their thyroid being blocked by Lugol's solution $(120 \mathrm{mg}$ in $100 \mathrm{ml}$ drinking water) 3 days before were randomly divided into the following 3 groups: group A $(n=10)$ of dual-targeting treatment received iv injections of CA4P $(10 \mathrm{mg} / \mathrm{kg})$ and, $24 \mathrm{~h}$ later, ${ }^{131} \mathrm{I}-\mathrm{Hyp}(300 \mathrm{MBq} / \mathrm{kg})$; group $\mathrm{B}(\mathrm{n}=10)$ of single targeting controls received $\mathrm{CA} 4 \mathrm{P}$ and solvent of ${ }^{131}$ I-Hyp solution; group $\mathrm{C}(\mathrm{n}=10)$ of dual vehicle controls only received iv injections of the solvents of the two drugs. Their health, activity level, and body weight (BW) were recorded daily. By intention, we did not include a control group that might have received the solvent of CA4P and 131I-Hyp for the following reasons: 1) previous studies have proven a lack of anticancer potency of ${ }^{131}$ I-Hyp alone [4, 9], 2) radioiodinated Hyp has been evidenced as a necrosis avid, instead of tumor specific, tracer, and 3) we wanted to minimize radiation hazard in this experiment. MRI and planar gamma scintigraphy were performed in vivo to monitor and quantify tumor volume and necrosis. Time to endpoint was recorded for survival analysis. At endpoint, all tumors were excised and weighed, and the tumor volumes were recorded using a measuring cylinder half filled with normal saline. Autoradiography and histopathology were performed for postmortem quantification and verification.

\section{Biodistribution study}

Mice were euthanized by anesthetic overdose at 24,72 , or $120 \mathrm{~h}$ to determine levels of ${ }^{131} \mathrm{I}-\mathrm{Hyp}$ in the blood, brain, heart, liver, spleen, kidneys, lungs, stomach, and activity ratios of necrotic/viable tumor tissues ( $n=3$ per time point). Tissues were harvested, drained of blood and weighted before radioactivity was measured with a 3-inch Nal (TI) gamma counter (Wallac Wizard, Turku, Finland). Standards representing the injected dose per animal were compared for calculation of the percentage injected dose per gram $(\% \mathrm{ID} / \mathrm{g})$ of tissues.

\section{Magnetic resonance imaging (MRI)}

MRI was performed using a clinical 3.0T MR magnet (Trio; Siemens, Erlangen, Germany) with a wrist coil for mouse studies. The mouse was gas-anesthesized with $2 \%$ isoflurane in the mixture of $20 \%$ oxygen and $80 \%$ room air, through a mask connected via a tube to a Harvard Apparatus system (Holliston, MA, USA), and placed supinely in a plastic holder. The penile vein of the mouse was cannulated for administration of contrast agent and drug. After positioning slices on scout images, T1-weighted (repetition time $=450 \mathrm{~ms}$; echo time $=12 \mathrm{~ms}$; field of view 
$=80 \times 63 \mathrm{~mm}^{2}$; imaging acquisition matrix $192 \times 192$; in plane resolution $0.42 \times 0.33 \mathrm{~mm}^{2}$; slice thickness $=$ $2.0 \mathrm{~mm}$; and voxel size $=0.4 \times 0.4 \times 2 \mathrm{~mm}$ ) and T2-weighted (repetition time $=4300 \mathrm{~ms}$; echo time $=$ $71 \mathrm{~ms}$ ) spin-echo multi-slice coronal images were acquired. Contrast enhanced T1-weighted (CE-T1W) images were obtained immediately after iv bolus of Gd-DOTA (Dotarem, Guerbet, France) at 0.2 $\mathrm{mmol} / \mathrm{kg}$.

\section{In vivo planar gamma scintigraphy}

Planar gamma scintigraphy was performed for Group A mice on day 3, 8, and 12 after ${ }^{131}$ I-Hyp injection using a dual-head gamma camera (Biad XLT 25; Trionix Research Laboratories, Twinsburg, Ohio) equiped with a pinhole high-energy collimator, which was immediately followed by a whole body computed tomography (CT, Biograph16, Siemens, Knoxville, TN, USA) scan for co-localization of the lesions on images of both modalities, which were aligned with rigid registration based on mutual information.

\section{Autoradiography}

Tumors and liver from group A mice at endpoint were quickly frozen in isopentane-liquid nitrogen and cut with a Cryotome (Microm HM 550, Walldorf,
Germany) into 10, 30 and 50 micrometer sections and were thaw-mounted on glass slides. Autoradiographs of these slides were obtained by $24-48 \mathrm{~h}$ exposure using a high-performance phosphor screen (super resolution screen; Canberra-Packard, Meriden, CT, USA). The images were analyzed using Optiquant software (Canberra-Packard). Relative tracer concentration in the necrotic tumor was estimated by regions of interest analysis for the necrotic and viable tumor as well as liver tissue on all autoradiographs.

\section{Histopathological procedures}

The frozen tissue slides from group A tumors were stained with hematoxylin and eosin (H\&E) to examine microscopically the tumor and intra-tumoral necrosis. Photomicrographs were obtained from an optical microscope (Axioskop; Zeiss, Oberkochen, Germany) with magnification at $\times 100$. For mice in group $\mathrm{B}$ and $\mathrm{C}$, tumors were excised at end point, fixed in $10 \%$ formalin, embedded in paraffin, and sectioned into 5 micrometer slices in the plane similar to that of in vivo MR images for H\&E staining. Digital photomicrographs were taken and compared with the corresponding MR images and autoradiographs.

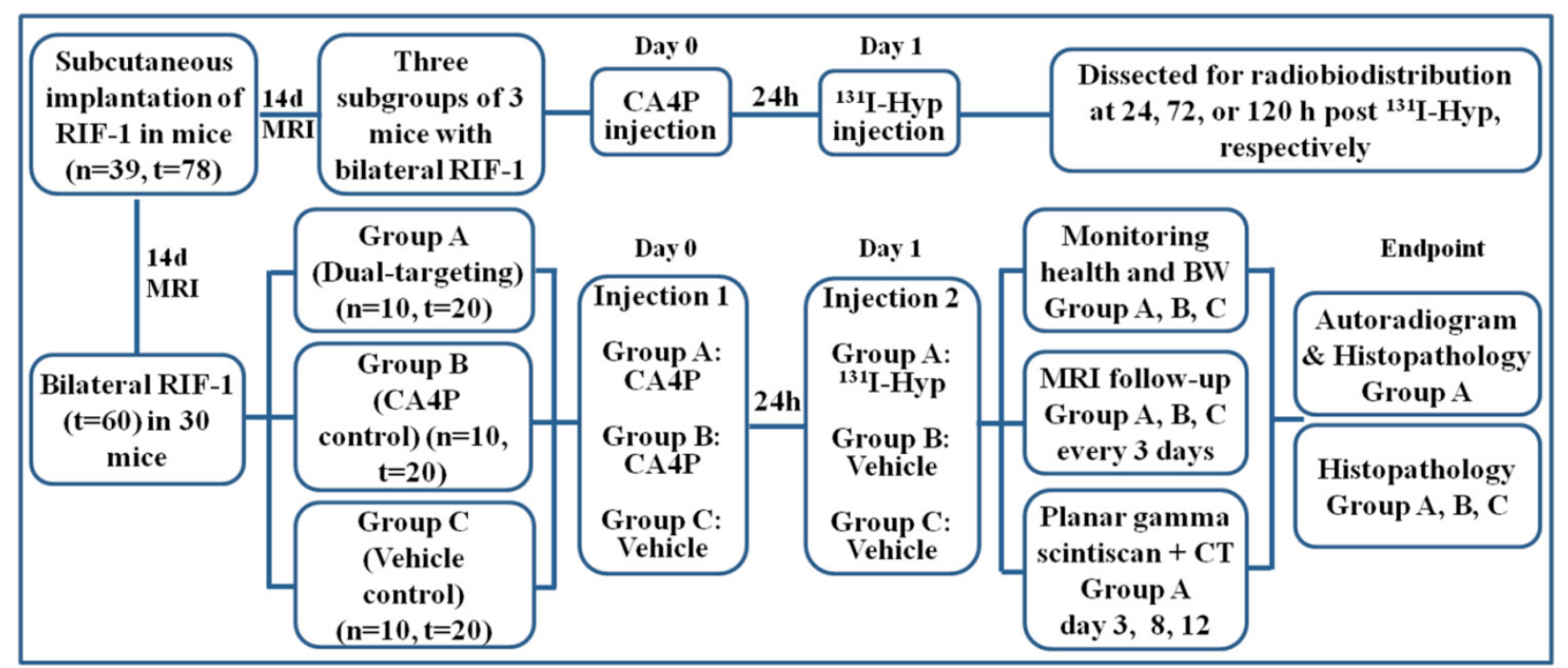

Figure 2. Flow diagram of experimental procedures in mice with bilateral subcutaneous RIF-I tumor xenografts (Hyp, Hypericin; n, number; t, tumor). 


\section{MR imaging analysis of tumoricidal effects}

Quantifications of tumor area were done by manually delineating the outline of the tumor mass on each T2-weighted MRI slice covering the whole tumor. Tumor volume was calculated using the equation: tumor volume $=\sum$ (tumor area on each slice $\times$ slice thickness). Tumor doubling time (DT) was calculated based on the formula: $\mathrm{DT}=\left(\mathrm{T}-\mathrm{T}_{0}\right) \times \log 2 /$ $\left(\log \mathrm{V}-\log \mathrm{V}_{0}\right)$, where $\left(\mathrm{T}-\mathrm{T}_{0}\right)$ indicates the time interval between two measurements, $\mathrm{V}_{0}$ and $\mathrm{V}$ denote the tumor volume at the two points of measurement [10]. The area of central nonenhancing region was delineated from CE-T1 images to estimate necrosis. Percentile necrosis ratios were defined as the volume of necrosis over that of entire tumor, i.e. necrosis ratio $=\sum$ (area of necrosis $\times$ slice thickness) / (area of whole tumor $\times$ slice thickness) $\times 100 \%$.

\section{Survival analyses}

For survival analysis, the primary endpoint was animal death. Standardized humane endpoint used to euthanize animals was failure to eat and drink for over 3 days and without any limb movement.

\section{Statistical analyses}

Numerical data were reported as mean \pm standard deviation. Statistical analysis was carried out with SPSS for Windows software package (version 16.0; SPSS, Chicago, IL, USA). For survival, Kaplan-Meier survival curves were made with $p$ value generated from log-rank test. For other comparisons, a one-way ANOVA was used to test differences among groups. A significant difference was considered for $p$ value less than 0.05 .

\section{Results}

\section{General conditions}

Almost all mice survived the surgery, anesthesia and imaging procedures without any drug administration-related deaths. One mouse died of overdose of anesthetics during tumor inoculation. The bilateral subcutaneous RIF-1 model was successfully established in 39 mice. Mice for survival study showed pallor, piloerection, weakness, less movement, stopping of eating and drinking at 2-3 days before death, with a rapid decrease of body weight.

\section{Biodistribution}

Relatively high uptake of ${ }^{131}$ I-Hyp was seen in the spleen, thyroid and lung one day after radioactive dosage (Table 1). Except the necrotic tumor, all organs and tissues revealed an obvious clearance of radioactivity within $72 \mathrm{~h}$. Systemic injection of ${ }^{131}$ I-Hyp resulted in distinctive high uptakes of radioactivity in necrotic tumor with $5.39 \pm 1.41,5.16 \pm 1.85$, and $4.74 \pm$ $0.90 \% \mathrm{ID} / \mathrm{g}$, as compared to $1.34 \pm 0.22,0.58 \pm 0.08$, $0.23 \pm 0.06 \% \mathrm{ID} / \mathrm{g}$ in the liver at 24,72 , and $120 \mathrm{~h}$, corresponding to a necrosis-to-liver activity ratio of $4.19 \pm 1.80,8.78 \pm 2.10$, and $22.23 \pm 9.20$, respectively.

\section{Survival}

Only one episode of SMSDTTS prolonged survival of the tumor bearing mice, the median survival in group A, B and C was 33, 22, and 21 days respectively (Fig. 3). Significant differences were found between group $\mathrm{A}$ and group $\mathrm{B}$ or $\mathrm{C}$ in survival curves $(p<0.001)$, no significant difference $(p=0.96)$ was shown in survival curves between group $\mathrm{B}$ and $\mathrm{C}$.

Table I. Biodistribution of ${ }^{|3|} \mid-H y p$ in radiation-induced fibrosarcomas-I (RIF-I) bearing SCID mice ( $n=3$ per time point) at 24, 72 or $120 \mathrm{~h}$ post injection: Data are expressed as percentage injected dose per gram of tissue (\%ID/g).

\begin{tabular}{llll}
\hline Organ & \multicolumn{2}{l}{ \% injected dose $/ \mathrm{g} \pm \mathrm{SD}$} & $72 \mathrm{~h}$ \\
\cline { 2 - 4 } & $24 \mathrm{~h}$ & $0.18 \pm 0.06$ & $0.02 \pm 0.00$ \\
\hline Blood & $0.75 \pm 0.26$ & $0.01 \pm 0.00$ & $0.01 \pm 0.00$ \\
Brain & $0.02 \pm 0.00$ & $3.12 \pm 6.13$ & $1.39 \pm 0.07$ \\
Thyroid & $4.38 \pm 2.44$ & $1.50 \pm 0.39$ & $0.36 \pm 0.12$ \\
Lungs & $2.81 \pm 0.31$ & $0.38 \pm 0.15$ & $0.14 \pm 0.04$ \\
Heart & $0.95 \pm 0.17$ & $0.58 \pm 0.08$ & $0.23 \pm 0.06$ \\
Liver & $1.34 \pm 0.22$ & $0.71 \pm 0.59$ & $0.51 \pm 0.17$ \\
Spleen & $2.79 \pm 1.20$ & $0.56 \pm 0.22$ & $0.07 \pm 0.03$ \\
Stomach & $1.32 \pm 0.55$ & $0.34 \pm 0.12$ & $0.08 \pm 0.04$ \\
Kidneys & $0.48 \pm 0.04$ & $0.96 \pm 0.35$ & $0.33 \pm 0.12$ \\
Viable Tumor & $2.18 \pm 1.19$ & $5.16 \pm 1.85$ & $4.74 \pm 0.90$ \\
Necrotic Tumor & $5.39 \pm 1.41$ & \\
\hline
\end{tabular}




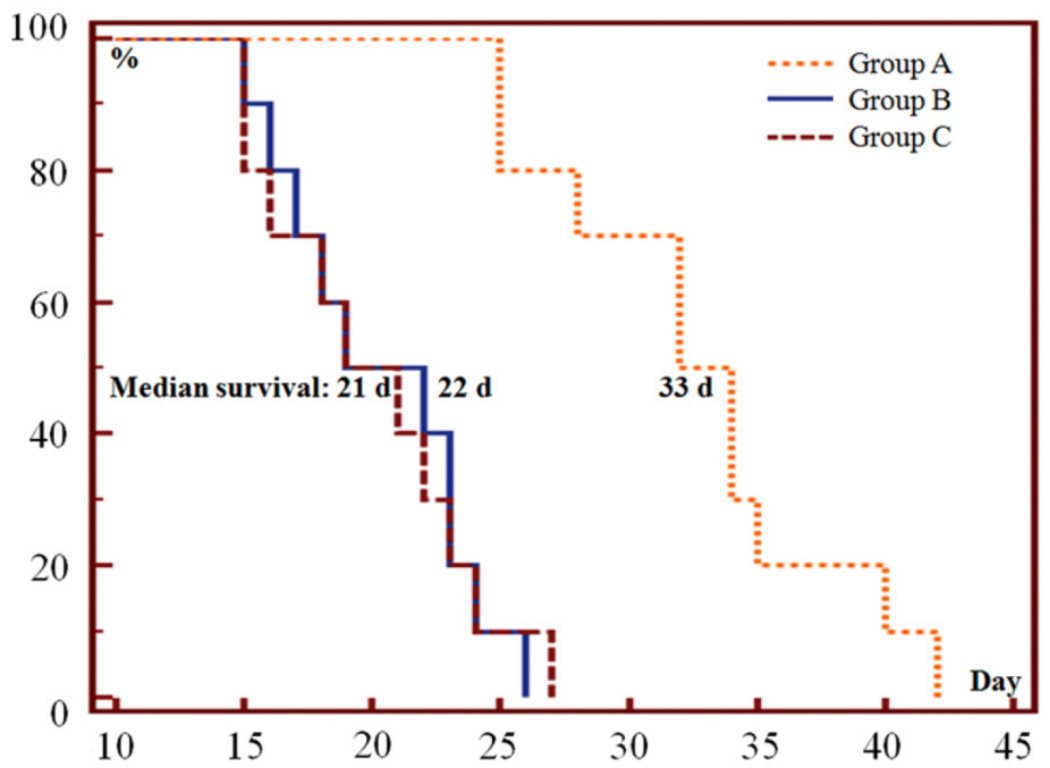

Figure 3. Kaplan-Meier survival curves show the survival probability (\%) of the 3 groups of mice. The median survival was 33 (range 25-42), 22 (range I5-26) and 2 I (range I5-27) days in group A, B and C respectively $(p<0.00$ I in A vs B or C; $P=0.96$ in $B$ vs $C)$.

\section{In vivo MRI and planar gamma scintigraphy}

Tumors at baseline appeared hyperintense on T2WI and slightly hypointense or isointense on T1WI. Contrast enhancement was observed on CE-T1WIs, suggesting hypervascularity of the subcutaneous RIF-1 mass. A nonenhanced central region surrounded by a thin rim enhancement presented on CE-T1WIs in group A and B after injection of CA4P, suggesting the presence of massive necrosis and minimum tumor residue, which differed from the tumors with spontaneous necrosis in group C (Fig.4).

On planar gamma scintigraphy, all bilateral tumors in group A were visible as hotspots from day 3 till 12 after the injection of ${ }^{131}$ I-Hyp, which became brighter and more focused as a function of time. Hotspots visualized in vivo on both sides of the upper flank corresponded exactly to the hypointense core of the tumor on CE-T1WI and the tumor sites in gross photography (Fig. 5).

\section{Tumor volume, tumor DT and necrosis ratio}

Tumor volumes and the corresponding necrosis ratio on day $0,3,6,9,12$, and 15 are shown (Fig. 6). Tumor volumes at baseline were $0.49 \pm 0.20,0.53 \pm$ 0.18 , and $0.50 \pm 0.17 \mathrm{~cm}^{3}$ in group $\mathrm{A}, \mathrm{B}$, and $\mathrm{C}$ respectively $(p>0.05)$. On day 15 , mean tumor volumes in group B $\left(5.66 \pm 1.66 \mathrm{~cm}^{3}\right)$ and $\mathrm{C}\left(5.02 \pm 1.03 \mathrm{~cm}^{3}\right)$ were about 2.5-2.8 times that of group A $\left(2.01 \pm 0.89 \mathrm{~cm}^{3}\right)$, with significantly prolonged tumor DT $(\mathrm{p}<0.01)$ in group A in comparison with group B and C $(7.8 \pm 2.8$ vs. $4.4 \pm 0.7$ and $4.5 \pm 0.5$ days, respectively). There was no significant difference $(p=0.083)$ between group $B$ and $C$ in either tumor volume or DT at the same time point.

Tumor necrosis as measured from CE-T1WI at baseline was negligible with median necrosis ratio lower than $10 \%$ (Fig. 6). After CA4P injection, a significantly increased necrosis ratio $(p<0.05)$ was obtained in group A and B. The ratio on day 1 showed no significant difference between group $A$ and $B$, but turned out to be $51 \pm 15 \%$ and $28 \pm 12 \%$ respectively on day $3(\mathrm{p}<0.01)$, indicating a progressively enlarged necrotic region in group $\mathrm{A}$, and a rapid tumor re-growth in group B. Significant differences $(p<0.05)$ were apparent between group A and group B or C from day 3 till day 15 . Necrosis ratio steadily increased in group $\mathrm{C}$ due to spontaneous occurrence (Fig. 6).

\section{Histopathological findings}

The borders between necrotic and viable tumor cells are clearly presented on microscopy (Fig. 4). Necrosis occupied a large-area of the tumor mass at the endpoint in all groups. In group A, exposure of tumor cells to ${ }^{131} \mathrm{I}$-radiation caused marked cell death in RIF-1 tumors, characterized by extensive and thorough intratumoral damage (Fig. 4). From the microscopic view of group B and C, nests of viable tumor cells and foci of spontaneous tumor necrosis coexisted, characterized by pyknotic, karyorrhectic nuclei, and clasmatosis (Fig. 4). 


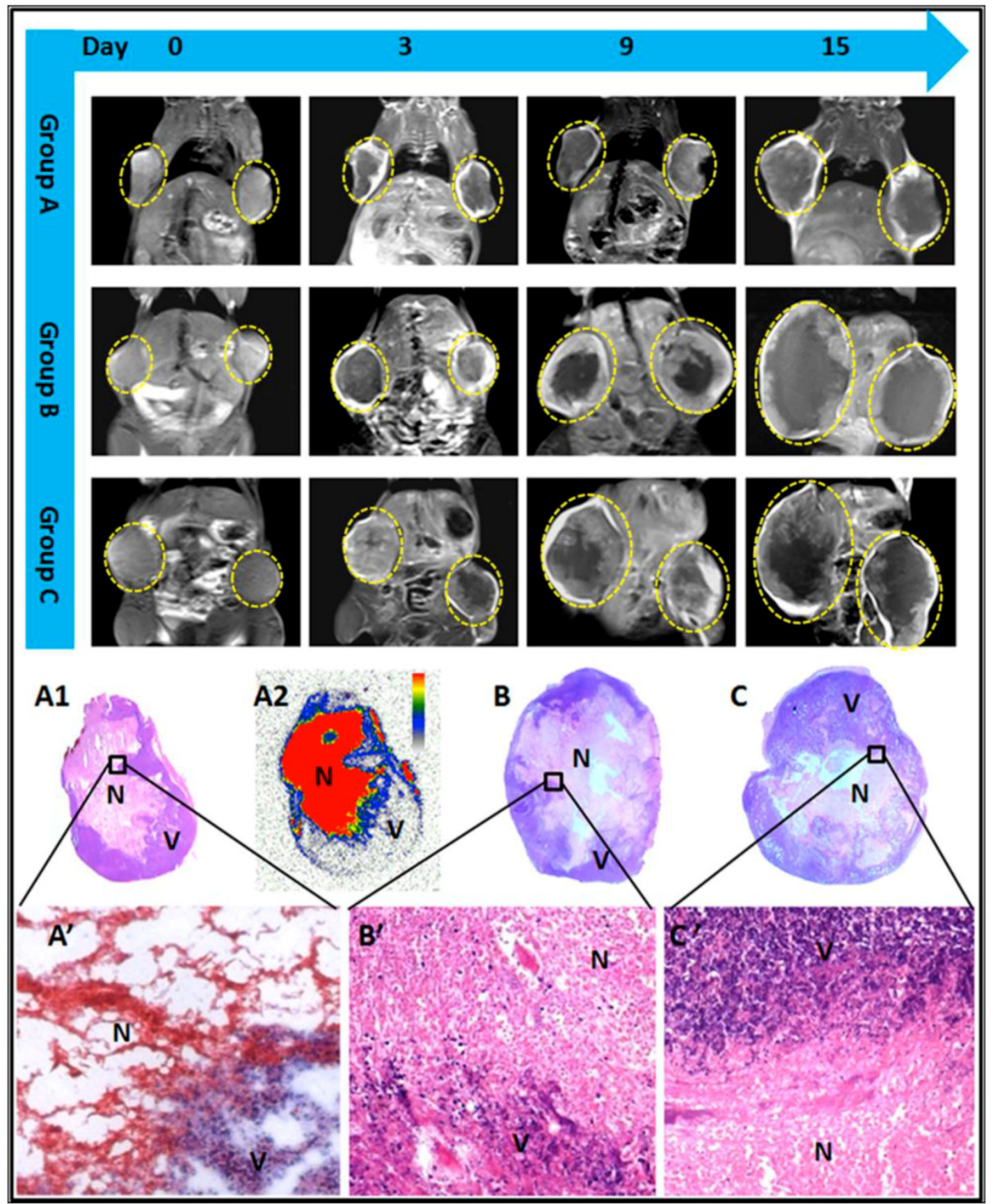

Figure 4. MR images of representative tumor bearing mice from the 3 groups, contrast enhanced TI (CE-TI) images on day 0,3 , 9, and 15 are shown. Tumors (delineated by dotted circles) in group $B$ and $C$ grew much faster than that of group $A$. Macroscopic photographs showed extensive central necrosis surrounded by viable tumor tissues in all groups of mice at endpoint ( $A$ I, B, C). Photomicroscopy (A', $\left.B^{\prime}, C^{\prime}\right)$ showed the interface between necrotic $(N)$ and viable $(V)$ tumor tissues in the 3 groups. The corresponding autoradiography $(A 2)$ at endpoint presented an extremely high uptake of radioactivity inside the tumoral necrosis induced by CA4P injection. 


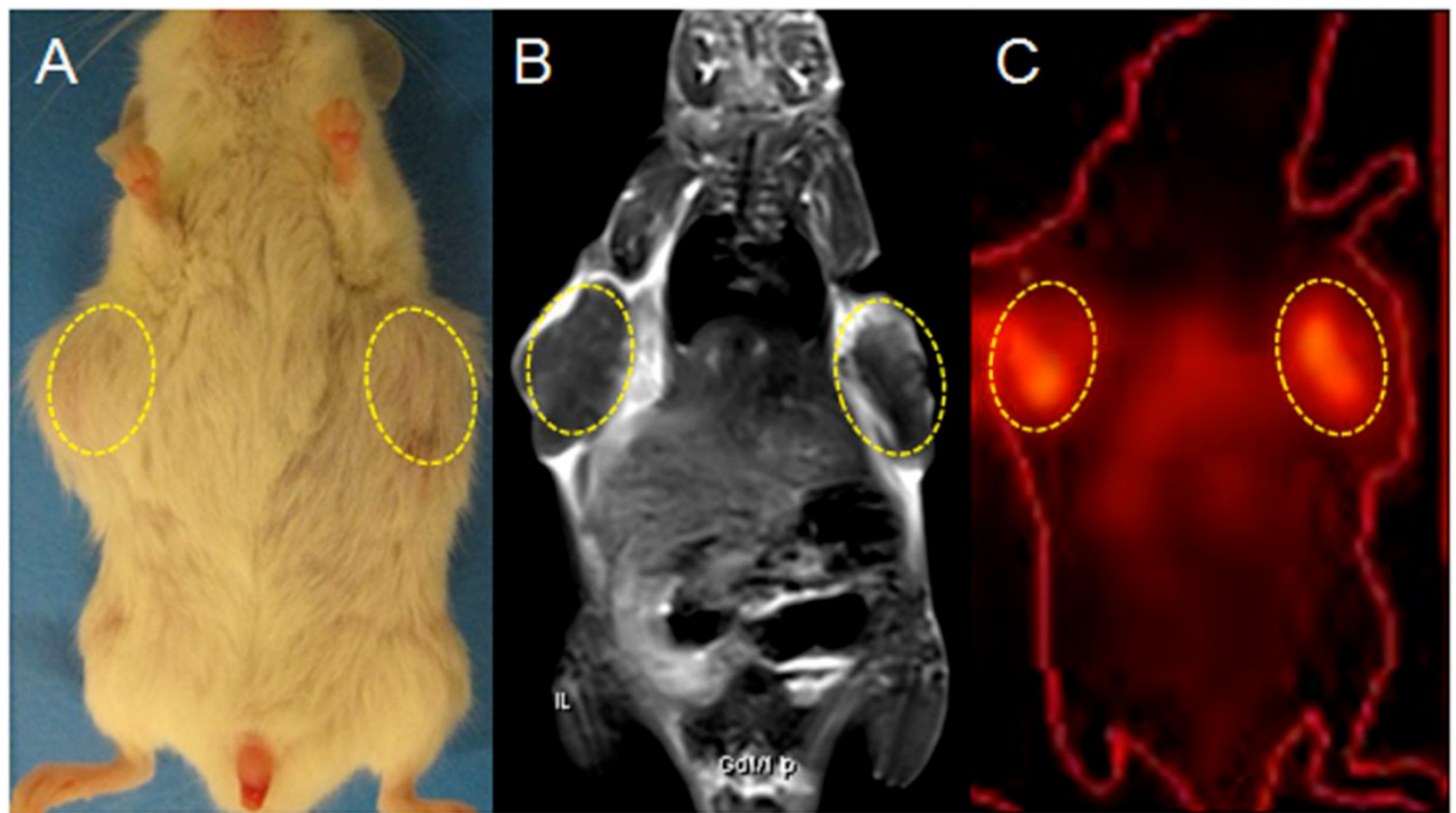

Figure 5. Gross photograph, CE-TI MRI and CT-coregistered planar scintigraphy ( $\mathrm{Y}$-scan) of a tumor bearing mouse in group $\mathrm{A}$ on day 12 post $13 \mid \mathrm{I}-\mathrm{Hyp}(\mathrm{A}, \mathrm{B}, \mathrm{C})$ : planar scintigraphy show a persistent hyperactivity mainly inside the tumor necrotic core (dotted circles). The hotspot imaging $(C)$ corresponds well with tumors on gross photograph (A), and the hypointense intratumoral region on CE-TIWI (B).

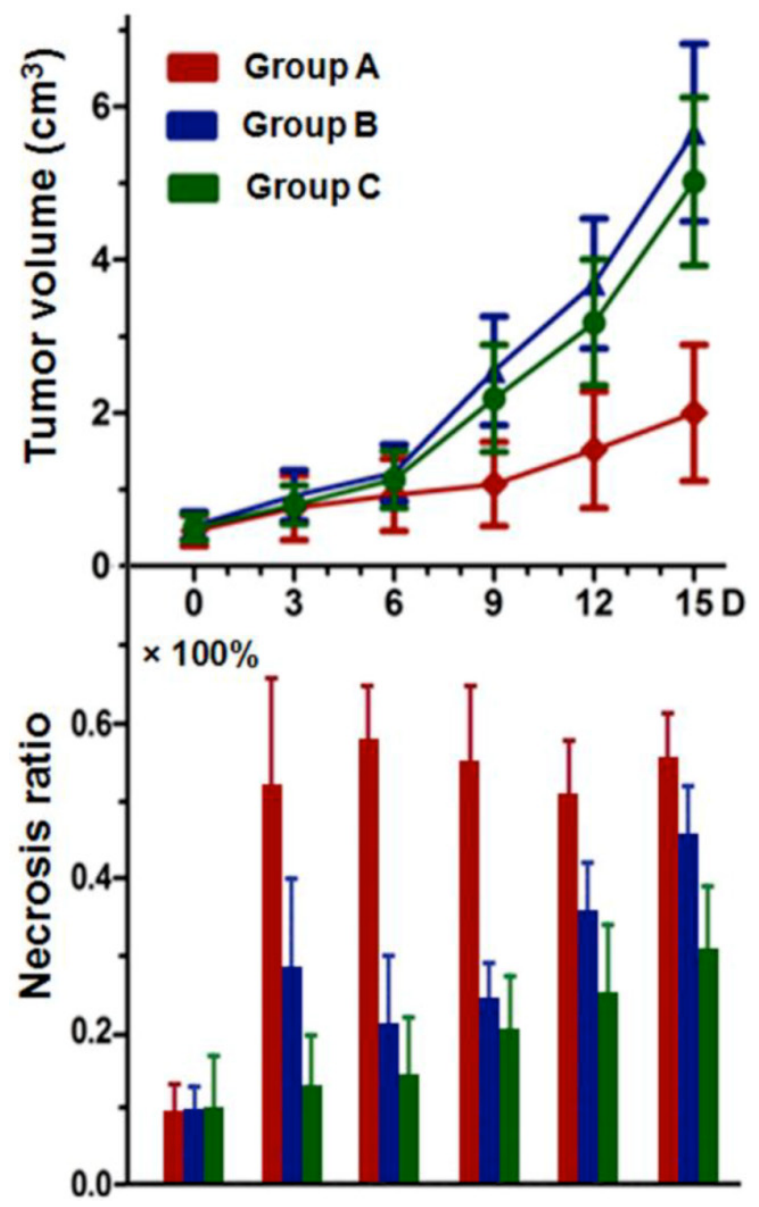

Figure 6. Tumor growth curve and corresponding necrosis ratios at baseline, and on day 3, 6, 9, I2, and I5 post-therapy: significant difference of tumor volume in group $A$ was found compared with that of group $B$ and $C(p<0.01)$ from day 6 on. No significant difference was found between group $B$ and $C$ at the same time point within follow-up of 15 days. Necrosis ratio measured from MRI presented a significant difference $(p<0.05)$ between each two of the studied groups (group A, B, and C) from day 3 on.

\section{Autoradiography}

Group A tumors revealed a distinct central aggregation of radioactivity at endpoint (Fig. 4). Intra-individually, as newly developed tumor tissue contained little radioactivity, necrotic to viable tumor activity ratio was extremely high ( $>100$ fold). A necrotic tumor to liver activity ratio of $24.0 \pm 2.5$ was obtained from the autoradiogram, consistent with gamma counting data (Table 1 ).

\section{Tumor weight and volume measurements at endpoint}

At endpoint, net weight of tumor was averaged as $3.3 \pm 1.9,6.2 \pm 2.1$, and $5.8 \pm 3.3 \mathrm{~g}$ for group $\mathrm{A}, \mathrm{B}$ and $\mathrm{C}$, respectively. Tumor weight in group A was found significantly lower in comparison with the other 2 groups $(\mathrm{p}<0.01)$. Tumor volume $\left(\mathrm{cm}^{3}\right)$ measured with the cylinder method approximated to the tumor weight (g) measured by the scale, suggesting a tumor density of about $1.0 \mathrm{~g} / \mathrm{cm}^{3}$. Meanwhile, the results 
were consistent with tumor volumes derived from the MRI, indicating a reliability of tumor volume measurement and calculation by in vivo MRI.

\section{Tendency of BW}

BW at baseline in group A, B and C was $26.9 \pm$ $1.0,27.0 \pm 2.5$, and $26.7 \pm 3.5$ g respectively without significant difference between groups. Mice in all groups presented an obvious tendency of gaining weight along with tumor growth. The corresponding BW on day 15 was $26.5 \pm 3.4,32.4 \pm 3.5$, and $32.0 \pm 2.2$ $\mathrm{g}$, with a significant increase in groups $\mathrm{B}$ and $\mathrm{C}$ $(p<0.01)$, relative to the small change in group $A$. However, the BW in group A reached $31.0 \pm 4.7 \mathrm{~g}$ on day 21 , which was also significantly increased compared with that at baseline $(\mathrm{p}<0.01)$. All mice demonstrated a rapid loss of BW from 3-5 days before death.

\section{Toxicity aspects}

Overall toxicity due to the treatment was minimal. Pallor, ascites, aggression, restlessness, piloerection, weakness, tremors, diarrhea, and loss of hair or appetite did not occur in groups B and C till day 14 or 5 days before death. Mice in group A revealed a visible fatigue and loss of appetite shortly after ${ }^{131} \mathrm{I}-\mathrm{Hyp}$ injection as proven by less movement and transitory weight loss, but these symptoms were only transient and disappeared within 3-5 days. No other signs of drug-related toxicity were shown. No specific toxic effects and obvious organ damage were found at visual inspection and microscopic observation of visceral organs.

\section{Discussion}

Hyp can be efficiently and reproducibly labeled with ${ }^{131} \mathrm{I}$ at a higher conjugation rate by Iodogen coating method as compared with the previously reported electrophilic substitution reaction [11,12]. Quantitative ex vivo gamma counting of radioactivity in dissected organs revealed a progressive clearance of ${ }^{131}$ I-Hyp from almost all organs such as blood, spleen and normal liver, but a sustained increasing uptake in tumor necrosis, resulting in a continuous increase in necrosis-to-liver activity ratio over $120 \mathrm{~h}$. This is in line with the earlier biodistribution data in rats [4]. As described previously $[13,14]$, radioiodinated Hyp is mainly taken up by the liver and quickly eliminated via the bile with a short biological half-life, which minimizes the hepatobiliary injuries and prevents the risk of renal damage as often seen with radioiodinated antibodies or peptides. Relatively high radioactivity in the lung and spleen at $24 \mathrm{~h}$ suggests possible aggregation of Hyp [11-15]. Considerable radioactivity in thyroid indicated some extent of in vivo deiodination of ${ }^{131}$ I-Hyp. Although gamma counting already indicated high radioactivity in necrotic tumor, the true results might be even better as it was difficult to distinguish pure necrosis from the tumor when sampling. Besides, radioactivity inside the necrosis was inhomogeneous due to the relatively poor blood perfusion. Both caused an averaged readout.

The concentration of ${ }^{131} \mathrm{I}-\mathrm{Hyp}$ in tumor necrosis was found a few orders of magnitude higher than that of a monoclonal antibody $(\mathrm{mAb})$ with a lengthy local retention over 3 weeks relative to 3 days with mAbs [16]. Consequently, the cumulative radiation dose in the tumor was estimated at about 100 times of the necessity for therapeutic response in most malignancies [4]. This explains the observed striking anticancer effects and significantly prolonged survival after only one treatment with SMSDTTS. Prominent therapeutic efficacies with SMSDTTS have also been evidenced by the significantly inhibited tumor volume growth and prolonged tumor DT. Meanwhile, regardless of the high radiation dose, SMSDTTS was well tolerated by all animals without the side effects commonly seen with other chemo- or radiotherapies. Unlike the previous studies using immunocompetent rodent models with tumor allografts [4-6], SCID mice have both dysfunctional innate immunity and lack of adaptive immunity. The preference to SCID mice in this study was based on the rationale that any anticancer efficacy could be attributable only to the drugs instead of natural immunity.

Despite the relatively poor spatial resolution of planar gamma scintigraphy, ${ }^{131}$ I-Hyp allowed long term imaging of tumor necrosis with high sensitivity for tumor detection, which to our knowledge has not been reported in the literature. Hotspot images of planar scintigraphy on day 12 indicated a very high accumulation of ${ }^{131}$ I-Hyp exclusively in the necrosis of the bilateral RIF-1 tumors. This finding not only supports the strong diagnostic capacity of ${ }^{131}$ I-Hyp for detecting sporadic malignancies over a relatively long period after injection, but also indicates a good stability of radioiodinated Hyp. Upon systemic injection, whole-body gamma scintigraphy may allow monitoring any primary or metastatic lesions, following up the therapeutic progress, and helping assess the patient prognosis.

The consistently high necrosis ratio in group A derived from in vivo MRI suggests a significant effect of ${ }^{131} \mathrm{I}-\mathrm{Hyp}$ to prevent rapid tumor re-growth or neo-angiogenesis as seen in group $\mathrm{B}$ and $\mathrm{C}$. The $\mathrm{BW}$ in all mice increased within a certain period along with the tumor volume increase. BW decreased only at the very end stage when the mice stopped eating and 
drinking. The net increment of tumor weight surpassed that of the gross BW at end stage and may suggest the presence of cachexia.

Tumors may become phenotypically more aggressive due to hypoxia following CA4P injection. With the regulation of hypoxia inducible factor $1 \mathrm{a}$ (HIF-1a), expression of angiogenic gene is activated to elevate the level of vascular endothelial growth factor, and endothelial progenitor cells are increased, which subsequently promote tumor neovascularization $[17,18]$. This may explain the lack of tumor inhibitory effect in group B comparing with $\mathrm{C}$. Therefore, the secondary targeting attack with ${ }^{131} \mathrm{I}-\mathrm{Hyp}$ proves compulsory in SMSDTTS for the desired complementary and synergistic anticancer outcomes.

Through the sequential use of VDA and the necrosis targeting radiocompound ${ }^{131}$ I-Hyp, a number of interactions might occur with the SMSDTTS. The first is the time coordination between the two drug administrations. Injection of the VDA should occur prior to ${ }^{131} \mathrm{I}-\mathrm{Hyp}$ injection to ensure the successful creation of tumor necrosis as the target for the latter agent. A 24h interval between VDA and ${ }^{131}$ I-Hyp may allow for a partial recovery of tumor vascularization and might provide better target accessibility to ${ }^{131}$ I-Hyp [19]. The second is the spatial complementation. The VDA kills the tumor from the inside out, leaving residual tumor cells mainly on the outer rim supplied by surrounding normal blood vessels, which facilitates 131 I-Hyp accumulated in the necrotic tissue bordering the viable rim to kill the remaining tumor cells. Such inherent and distinct mechanisms of the two drugs form a perfectly coupled attack on the tumor. The third is the synergistic effect. A recent study [5] showed a typical thickness of the remaining viable tumor to be about 20-100 $\mu \mathrm{m}$ following CA4P treatment at $10 \mathrm{mg} / \mathrm{kg}$, which is considered within the irradiation range of the beta particles emitted by ${ }^{131}$ I. Meanwhile, those viable and oxygen-saturated cells are rapidly proliferating and more sensitive to radiotherapy [20]. The highly specific and long-term accumulation of the energetic ${ }^{131}$ I-Hyp creates a constant and harsh microenvironment, which would eradicate any cells within the beta particle penetrated area by the 131I crossfire radiation effect, leading to a highly synergistic therapeutic efficacy. The last is the independent cytotoxic effects. Previous studies have also suggested that both VDA and 131I-Hyp may allow their direct cytotoxic effects onto different types of tumor cells via different mechanisms of actions [21-23].

The prominent theranostic potency, good in vivo tolerance, less-toxic features, and compatibility to multifocal or different tumor types [4-6] as demonstrated in this study would make SMSDTTS a natural, versatile and simple strategy that allows higher targeted radiation delivery and greater probability of complete tumor response. Meanwhile, as an ideal candidate for further preclinical exploration and future clinical application, optimal retention of therapeutic payload of ${ }^{131} \mathrm{I}-\mathrm{Hyp}$ within the tumor site has been proven in this and previous studies $[4,5,9]$. Limited radiotoxicity of Iodine-131 has been well documented by its clinical applications for decades. Nonor negligible chemotoxicities of iodinated-Hyp as well as involved reagents also have been shown recently $[24,25]$. Thus, a new horizon of improved cancer treatability, detectability or even curability can be anticipated with further development of SMSDTTS [26].

\section{Abbreviations}

BW: body weight; CA4P: combretastatin A4 phosphate; CE-T1W: contrast enhanced T1-weighted; CT: computed tomography; DT: doubling time; H\&E: hematoxylin and eosin; HIF-1a: hypoxia inducible factor-1a Hyp: hypericin; ${ }^{131} \mathrm{I}-\mathrm{Hyp}$ : ${ }^{131} \mathrm{I}$-iodohypericin; mAb: monoclonal antibody; MRI: magnetic resonance imaging; NAC: necrosis-avid compound; PBS: phosphate buffered saline; R1: rhabdomyosarcoma-1; RIF-1: radiation-induced fibrosarcoma-1; SCID: severe combined immunodeficiency; SMSDTTS: small-molecular sequential dual-targeting theragnostic strategy; VDA: vascular disrupting agents.

\section{Acknowledgments}

This work was partially supported by the grants awarded by FWO Vlaanderen ZWAP/05/018; Geconcerteerde Onderzoeksactie of the Flemish Government, OT project (OT/06/70); the KU Leuven Molecular Small Animal Imaging Center MoSAIC (KUL EF/05/08); the center of excellence In vivo Molecular Imaging Research (IMIR) of KU Leuven; the IWT SBO 'Imagine' (SBO80017), the National Natural Science Foundation of China (81071828), Jiangsu Province Natural Science Foundation (BK2010594) and a EU project Asia-Link CfP 2006-EuropeAid/123738/C/ACT/Multi-Proposal No. 128-498/111. The corresponding author Yicheng $\mathrm{Ni}$ is currently a Bayer Lecture Chair holder.

\section{Competing Interests}

The authors have declared that no competing interest exists.

\section{References}

1. Paget S. The distribution of secondary growths in cancer of the breast. Lancet. 1889; 1:571-573.

2. Kuonen F, Secondini C, Ruegg C. Molecular Pathways: Emerging pathways mediating growth, invasion and metastasis of tumors progressing 
in an irradiated microenvironment. Clin Cancer Res. 2012;18(19):5196-202.

3. Ni Y, Van de Putte M, de Witte P, et al. Targeted radiotherapy. European patent EP2219683B1, Date of publication 2012; Priority: 26.11.2007.

4. Li J, Sun Z, Zhang J, et al. A dual-targeting anticancer approach: soil and seed principle. Radiology. 2011; 260: 799-807.

5. Li J, Cona MM, Chen F, et al. Exploring Theranostic Potentials of Radioiodinated Hypericin in Rodent Necrosis Models. Theranostics. 2012; 2: 1010-1019.

6. Li J, Chen F, Cona MM, et al. A review on various targeted anticancer therapies. Target Oncol. 2012; 7: 69-85.

7. Brown J, Giaccia A. The unique physiology of solid tumors: opportunities (and problems) for cancer therapy. Cancer Res. 1998; 58: 1408-16.

8. Dark G, Hill S, Prise V, et al. Combretastatin A-4 agent that displays potent and selective toxicity toward tumor vasculature. Cancer Res. 1997; 57: 1829-34.

9. Van de Putte M, Marysael T, Fonge H, et al. Radiolabeled iodohypericin as tumor necrosis avid tracer: diagnostic and therapeutic potential. Int J Cancer. 2012; 131(2): 129-37.

10. Ozono S, Miyao N, Igarashi T, et al. Tumor doubling time of renal cell carcinoma measured by CT: collaboration of Japanese Society of Renal Cancer. Jpn J Clin Oncol.2004; 34:82-5.

11. Fonge $\mathrm{H}$, Van de Putte M, Huyghe D, et al. Evaluation of tumor affinity of mono-[(123)I]iodohypericin and mono-[(223)I]iodoprotohypericin in a mouse model with a RIF-1 tumor. Contrast Media Mol Imaging. 2007; 2: 113-9.

12. Fonge $\mathrm{H}$, Vunckx $\mathrm{K}$, Wang $\mathrm{H}$, et al. Non-invasive detection and quantification of acute myocardial infarction in rabbits using mono-[123I]iodohypericin microSPECT. Eur Heart J. 2008; 29: 260-9.

13. Van de Putte M, Wang H, Chen F, de Witte P, Ni Y. Hypericin as a marker for determination of tissue viability after intratumoral ethanol injection in a murine liver tumor model. Acad Radiol. 2008; 15:107-13.

14. Bormans G, Huyghe D, Christiaen A, et al. Preparation, analysis and biodistribution in mice of iodine-123 labelled derivatives of hypericin. J. Labelled Compd. Radiopharm.2004; 47: 191-198.

15. Ni Y, Huyghe D, Verbeke $\mathrm{K}$, et al. First preclinical evaluation of mono-[123I]iodohypericin as a necrosis avid tracer agent. Eur J Nucl Med Mol. 2006; 33:595-601.

16. Ni Y. MR Contrast agents for cardiac imaging. In: Bogaert J, Dymarkowski S, Taylor A, Muthurangu V, eds. Clinical Cardiac MRI. Berlin Heidelberg: Springer-Verlag. 2012:31-51.

17. Shaked Y, Kerbel R. Antiangiogenic strategies on defense: on the possibility of blocking rebounds by the tumor vasculature after chemotherapy. Cancer Res.2007; 67:7055-8.

18. Koljenovic S, Choo-Smith L, Bakker Schut T, et al. Discriminating vital tumor from necrotic tissue in human glioblastoma tissue samples by raman spectroscopy. Laboratory Investigation.2002; 82: 1265-1277.

19. Marysael T, Ni Y, Lerut E, de Witte P. Influence of the vascular damaging agents DMXAA and ZD6126 on hypericin distribution and accumulation in RIF-1 tumors. J Cancer Res Clin Oncol.2011; 137:1619-27.

20. Tozer G, Kanthou C, Lewis G, et al. Tumor vascular disrupting agents: combating treatment resistance. Br J Radiol. 2008; 81:S12-20.

21. Dziba J, Marcinek R, Venkataraman G, Robinson J, Ain K. Combretastatin A4 phosphate has primary antineoplastic activity against human anaplastic thyroid carcinoma cell lines and xenograft tumors. Thyroid. 2002; 12: 1063-70.

22. Dong D, Ko B, Baumeister P, et al. Vascular targeting and antiangiogenesis agents induce drug resistance effector GRP78 within the tumor microenvironment. Cancer Res.2005; 65: 5785-91.

23. Chen B, Roskams T, de Witte P. Enhancing the antitumoral effect of hypericin-mediated photodynamic therapy by hyperthermia. Lasers Surg Med. 2002; 31:158-63.

24. Li J, Cona MM, Feng Y, et al. A Single-dose Toxicity Study in Mice on Non-radioactive Iodinated Hypericin for a Targeted Anticancer Therapy. Acta Pharmacologica Sinica. 2012; 33:1549-56.

25. Cona MM, Li JJ, Chen F, et al. A safety study on single intravenous dose of tetrachloro-diphenyl glycoluril [Iodogen] dissolved in dimethyl sulfoxide [DMSO]. XENOBIOTICA. 2013; [Epub ahead of print].

26. Li J, Oyen R, Verbruggen A, Ni Y. Small Molecule Sequential Dual-Targeting Theragnostic Strategy (SMSDTTS): from Preclinical Experiments towards Possible Clinical Anticancer Applications. J Cancer. 2013 ; 4: 133-145. 